\title{
A NOTE ON A PROBABILISTIC DECOMPOSITION OF LINEAR DELTA MODULATOR OF A WIENER PROCESS
}

\author{
T. Koski
}

\section{Introduction}

Linear delta modulation (LDM) is a causal, recursive analog-to-digital (and vice versa) technique of data compression. The engineering work devoted to LDM and its modification is covered in [14]. Some of the research in the mathematical treatment of LDM is found in [5], [6], [13] and [17]. Further references e.g. to the works of T. Fine and J. Kiefer are found in [9].

This paper considers the LDM of a standard Wiener process (although some extensions are outlined). That this should be, in a certain sense, the proper way of tracking a time sampled Wiener process has been stated in [1]. Here we provide a probabilistic decomposition of the decoded process, by means of Ito's rule, viewing the decoded variable as a functional of the Wiener process. Other results on LDM of Wiener processes are found in [11].

The basic definitions of the LDM model are presented in Section 2. The main result, a probabilistic structure for the encoder, is given in Section 3 . It turns out that the encoder can be written as a sum of a function of the predicted reconstruction error and a stochastic integral. This shows that there is a certain martingale structure associated with the decoded process, the properties of which are studied in this section. It is quite obvious that the representation can be extended to other (diffusion) sources. Explicit results are given for the OrnsteinUhlenbeck case (see also [9]).

Section 4 contains an application of Malliavin's calculus on the predicted error part in the encoded process. It turns out that this part has a probability density with respect to the Lebesgue measure.

\section{Linear delta modulation of a Wiener process}

Let $(\Omega, \mathcal{F}, P)$ be a complete probability space and $w^{d}=\left\{w_{t_{i}}\right\}_{i=0}^{\infty}$ be a real (source) stochastic process defined on it (in fact $w^{d}$ is a time-sampled Brownian motion (B.M.)). Let $d>0$ and $0<c \leq 1$. The encoder of the LDM is defined by the predictor

$$
b_{i}=\operatorname{sgn}\left(w_{t_{i}}-c z_{t_{i-1}}\right)
$$


where we take

$$
\operatorname{sgn}(x)=\left\{\begin{aligned}
+1, & x>0 \\
0, & x=0 \\
-1, & x<0
\end{aligned}\right.
$$

In (2.1) the process $z^{d}=\left\{z_{t_{i}}\right\}_{i=0}^{\infty}$ is the decoded sequence of random variables that approximate $w^{d}$ and is recursively generated by means of the corrector

$$
z_{t_{i}}=c z_{t_{i-1}}+d b_{i}
$$

The engineering terms for (2.3) are "ideal integration" in case $c=1$ and "leaky integration" for $c<1$. Further, $d$ may be regarded as a quantization step. Solving (2.3) yields

$$
z_{t_{i}}=c^{i} z_{t_{0}}+d \sum_{j=1}^{i} c^{i-j} b_{j} .
$$

When dealing with LDM of B.M. it is natural to set $z_{t_{0}}=0$. Then it follows for the leaky integrator that

$$
\left|z_{t_{i}}\right| \leq \frac{d}{1-c}
$$

for every $t_{i}$. Regard now the random variables $w_{t_{i}}$ as samples at deterministic times of an underlying B.M. $w=\left\{w_{t} \mid t \geq 0\right\}$ defined on $(\Omega, \mathcal{F}, P)$. Let

$$
\mathcal{F}_{t}=\sigma\left(w_{s} \mid 0 \leq s \leq t\right)
$$

designate the sigma-algebra generated by the process $w$ up to time $t$. It is evident by construction that $z_{t_{i}}$ cannot converge anywhere on $\Omega$ as $i \rightarrow \infty$, if $w$ is the source process. Hence it follows that

2.1. Proposition. Let $w^{d}$ be a sampled B.M. Then $\left(z_{t_{i}}, \mathcal{F}_{t_{i}}\right)_{i=0}^{\infty}$ cannot be an asymptotic martingale if $0<c<1$.

The proof follows immediately by Proposition 2.2 in [4] in view of (2.5). In particular this means that $\left(z_{t_{i}}, \mathcal{F}_{t_{i}}\right)_{i=0}^{\infty}$ cannot be a martingale (sub- or quasi). It can be seen that a similar conclusion holds for the ideal integrator, too. However, we shall discover that a martingale structure is associated with LDM of B.M.

The basic point of departure is the following observation: (2.5) entails

$$
E\left|z_{t_{i}}\right|^{2} \leq d^{2} /(1-c)^{2}
$$

for leaky integration. Hence any $z_{t_{i}}$ may be viewed as a square integrable functional of $w$ up to $t_{i}$. As is well established (c.f. [2] and [10], Theorem 5.6), any such functional of the Wiener process $w^{t_{i}}=\left(w_{t}, \mathcal{F}_{t}\right)_{0 \leq t \leq t_{i}}$ can be written as a sum of an random variable and a stochastic integral.

Note also that $E\left|z_{t_{i}}\right|^{2} \leq c_{i}<\infty$ for some finite constant $c_{i}$ if ideal integration is considered, since any $z_{t_{i}}$ has a range consisting of some finite, countable number of values. Consequently we need not consider separately the two cases in Propositions 3.1 and 3.4. 


\section{The representation of LDM by means of a stochastic integral}

First we make some elementary observations about the function

$$
V\left(t, x ; z, t_{i}\right)=\int_{-\infty}^{\infty} k\left(t, x-y ; t_{i}\right) \operatorname{sgn}(y-z) d y
$$

where $z \in R$ and

$$
k\left(t, x ; t_{i}\right)= \begin{cases}\frac{e^{-x^{2} / 4\left(t_{i}-t\right)}}{\sqrt{4 \pi\left(t_{i}-t\right)}} & 0 \leq t<t_{i} \\ 0 & t=t_{i}\end{cases}
$$

is the heat kernel. The classical change of variable ([15], p. 32) $y \rightarrow r$

$$
r=(y-x) \cdot\left(4\left(t_{i}-t\right)\right)^{-1 / 2}
$$

gives after an elementary calculation that for $\Theta=(z-x) / \sqrt{4 \pi\left(t_{i}-t\right)}$

$$
V\left(t, x ; z, t_{i}\right)=\operatorname{erfc}(\Theta)-1
$$

where erfc is the complementary error function

$$
\operatorname{erfc}(x)=\frac{2}{\sqrt{\pi}} \int_{x}^{\infty} e^{-r^{2}} d r
$$

([15], p. 10). By quick separate consideration of the three cases $\operatorname{sgn}(x-z)= \pm 1$ or 0 one sees that

$$
\lim _{t \uparrow t_{i}} V\left(t, x ; z, t_{i}\right)=\operatorname{sgn}(x-z)
$$

for every $x$ and $z$. (This is more than can be expected in the general case, c.f. [15], Chapter VII, and is the reason for the definition (2.2)). It is also a straightforward matter to check that the function $(t, x) \rightarrow V\left(t, x ; z, t_{i}\right)$ solves (for fixed $t_{i}$ and $z$ ) the backward heat equation

$$
V_{t}^{\prime}(t, x)+\frac{1}{2} V_{x x}^{\prime \prime}(t, x)=0 \quad \text { for } \quad(t, x) \in\left[0, t_{i}[\times]-\infty, \infty[\right.
$$

where $V_{t}^{\prime}$ and $V_{x x}^{\prime \prime}$ denote partial differentiation. The boundary values are those in (3.6). The basic rules of stochastic calculus provide the link between (3.1)-(3.7) and the LDM for $w^{d}$ (c.f. [3]). 
3.1. Proposition. Let $w^{d}$ consist of discrete time samples of a standard B.M. $w$ and let $z^{d}$ be the corresponding encoded process (evolving according to (2.3)). Then the equality

$$
\begin{gathered}
b_{i}=\operatorname{sgn}\left(w_{t_{i}}-c z_{t_{i-1}}\right)=\operatorname{erfc}\left(\frac{c z_{t_{i-1}}-w_{t_{i-1}}}{\sqrt{4 \pi\left(t_{i}-t_{i-1}\right)}}\right)-1 \\
+\int_{t_{i-1}}^{t_{i}} \frac{1}{\sqrt{t_{i}-s}} e^{-\left(c z_{t_{i-1}}-w_{s}\right)^{2} / 4 \pi\left(t_{i}-s\right)} d w_{s}
\end{gathered}
$$

holds, $P$-almost surely, for any $i$.

Proof. In view of (3.7) a formal application of Ito's differentiation rule yields

$$
\begin{aligned}
V\left(t_{i}, w_{t_{i}} ; c z_{t_{i-1}}, t_{i}\right)-V\left(t_{i-1}, w_{t_{i-1}} ;\right. & \left.c z_{t_{i-1}}, t_{i}\right)= \\
& =\int_{t_{i-1}}^{t_{i}} V_{x}^{\prime}\left(s, w_{s} ; c z_{t_{i-1}}, t_{i}\right) d w_{s} .
\end{aligned}
$$

It may be noted that $V_{x}^{\prime}\left(s, w_{s} ; c z_{t_{i-1}}, t_{i}\right)$ is $\mathcal{F}_{s}$-measurable, as it should be, and

$$
\int_{t_{i-1}}^{t_{i}} E\left[V_{x}^{\prime}\left(s, w_{s} ; c z_{t_{i-1}}, t_{i}\right)\right]^{2} d s<\infty .
$$

In order to check (3.10) we observe that

$$
V_{x}^{\prime}\left(s, x ; z, t_{i}\right)= \begin{cases}\frac{e^{-(z-x)^{2} / 4 \pi\left(t_{i}-s\right)}}{\sqrt{t_{i}-s}} & s<t_{i}, \\ 0 & s=t_{i} .\end{cases}
$$

which is in its turn readily established e.g. by differentiating (3.1) and performing the change of variable (3.3) in the resulting integral.

Here

$$
\begin{aligned}
E\left[V_{x}^{\prime}\left(s, w_{s} ; c z_{t_{i-1}}, t_{i}\right)\right]^{2} & =\frac{1}{t_{i}-s} E\left[e^{-\left(c z_{t_{i-1}}-w_{s}\right)^{2} / 2 \pi\left(t_{i}-s\right)}\right] \\
& =\frac{1}{t_{i}-s} E\left[E\left[e^{-\left(c z_{t_{i-1}}-w_{s}\right)^{2} / 2 \pi\left(t_{i}-s\right)} \mid \mathcal{F}_{t_{i-1}}\right]\right] .
\end{aligned}
$$

Given $\mathcal{F}_{t_{i-1}}$ the random variable $w_{s}$ has a normal distribution $N\left(w_{t_{i-1}} ; s\right)$ and $z_{t_{i-1}}$ has assumed a fixed value in some finite and countable set. Under these circumstances it can be calculated, in a straightforward manner, that for a constant $M$

$$
E\left[\left(e^{-\left(c z_{t_{i-1}}-w_{s}\right)^{2} / 2 \pi\left(t_{i}-s\right)}\right) \mid \mathcal{F}_{t_{i-1}}\right]=M \sqrt{t_{i}-s} E\left[e^{y_{s}(i)}\right]
$$


where $y_{s}(i)$ is a random variable which is easily seen to be almost surely uniformly bounded in $s \in\left[t_{i-1}, t_{i}\right]$. Hence

$$
\int_{t_{i-1}}^{t_{i}} V_{x}^{\prime}\left(s, w_{s} ; z_{t_{i-1}}, t_{i}\right) d w_{s}
$$

is a well defined stochastic integral in Ito's sense and (3.8) is valid. Let us now set

$$
\begin{aligned}
x_{i}(w) & =\int_{t_{i}-1}^{t_{i}}\left(t_{i}-s\right)^{-1 / 2} \exp \left(-\left(c z_{t_{i-1}}-w_{s}\right)^{2} / 4 \pi\left(t_{i}-s\right)\right) d w_{s} \\
\mathbf{F}_{t_{i}}(w) & =\left[\operatorname{erfc}\left(\frac{c z_{t_{i-1}}-w_{t_{i-1}}}{\sqrt{4 \pi\left(t_{i}-t_{i-1}\right)}}\right)-1\right]
\end{aligned}
$$

Evoking Proposition 3.1 and these notations in (2.4) yields the decomposition

\subsection{Corollary.}

$$
z_{t_{i}}=d c^{i} \sum_{j=1}^{i} c^{-j} \mathbf{F}_{t_{j}}(w)+d c^{i} \sum_{j=1}^{i} c^{-j} x_{j}(w) .
$$

The elementary properties of stochastic integrals as well as the fact that every $\mathbf{F}_{t_{i}}(w)$ is $\mathcal{F}_{t_{i-1}}$-measurable entail, by virtue of (3.15), that the conditional variance of $z_{t_{i}}$ equals

$$
E\left[\left(z_{t_{i}}-E\left[z_{t_{i}} \mid \mathcal{F}_{t_{i-1}}\right]\right)^{2} \mid \mathcal{F}_{t_{i-1}}\right]=E\left[x_{i}^{2}(w) \mid \mathcal{F}_{t_{i-1}}\right] .
$$

which would seem to indicate that some interesting information about the process $\left\{z_{t_{i}}\right\}_{i=0}^{\infty}$ could be obtained in terms of the properties of the martingale $\left(S_{i}, \mathcal{F}_{t_{i}}\right)_{i=0}^{\infty}$ where

$$
S_{i}=\sum_{j=1}^{i} x_{j}(w), \quad S_{0}=0
$$

For example we find the following using Theorem 2.15 in [7]:

3.3. Proposition. Let $\left\{z_{t_{i}}\right\}_{i=0}^{\infty}$ be the decoded sequence of the leaky integration LDM of B.M. Suppose that $\sum_{i=1}^{\infty} \sqrt{\left(t_{i}-t_{i-1}\right)}<\infty$. Then

$$
\sum_{i=1}^{\infty} E\left[x_{i}^{2}(w) \mid \mathcal{F}_{t_{i-1}}\right]<\infty
$$


almost surely, and

$$
\sum_{i=1}^{n} c^{n-i} x_{i}(w) \rightarrow 0
$$

almost surely, as $n \rightarrow \infty$.

The proof will be published elsewhere. This result is mostly of theoretical interest, since in practice $t_{i}-t_{i-1}$ is almost constant.

Suppose now that the process being linearly delta modulated is an OrnsteinUhlenbeck process (O.U.) (or Gauss-Markov process). Denoting the time samples by $\left\{x_{t_{i}}\right\}_{i=0}^{\infty}$ and letting $\left\{z_{t_{i}}\right\}_{i=0}^{\infty}$ denote the corresponding output of the decoder, we obtain by explicit calculation exactly similar to above (but somewhat more arduous) that

3.4. Proposition. Let the process $x=\left\{x_{t} \mid t \geq 0\right\}$ have the stochastic differential

$$
d x_{t}=-\mu x_{t} d t+d w_{t}, \quad x_{0}=0
$$

where $w$ is a standard B.M. and $\mu>0$.

Then

$$
\begin{aligned}
& \operatorname{sgn}\left(x_{t_{i}}-c z_{t_{i-1}}\right)=\operatorname{erfc}\left(\frac{\sqrt{\mu}\left(c z_{t_{i-1}}-x_{t_{i-1}} e^{-\mu\left(t_{i}-t_{i-1}\right)}\right)}{\left(1-e^{-2 \mu\left(t_{i}-t_{i-1}\right)}\right)^{1 / 2}}\right)-1 \\
& +\frac{2 \mu}{\sqrt{\pi}} \int_{t_{i-1}}^{t_{i}} \frac{e^{-\mu\left(t_{i}-s\right)}}{\sqrt{1-e^{-2 \mu\left(t_{i}-s\right)}}} \exp \left(-\mu \frac{\left(x_{s} e^{-\mu\left(t_{i}-s\right)}-c z_{t_{i-1}}\right)^{2}}{\left(1-e^{-2 \mu\left(t_{i}-s\right)}\right)}\right) d w_{s} .
\end{aligned}
$$

We omit the detailed proof but point out that the partial differential equation corresponding to (3.6)-(3.7) is in this case

$$
\begin{aligned}
& \left.V_{t}^{\prime}+\frac{1}{2} V_{x x}^{\prime \prime}-\mu x V_{x}^{\prime}=0 \text { for }(t, x) \in\right] 0, t_{i}[\times]-\infty, \infty[ \\
& \lim _{t \uparrow t_{i}} V\left(t, x ; z, t_{i}\right)=\operatorname{sgn}(x-z) .
\end{aligned}
$$

The statement of the counterpart of Corollary 3.2 is obvious. Further analysis of the Ornstein-Uhlenbeck case using this representation in order to derive results like in [6] and [13] is in progress, see [9].

The formula (3.21) provides some further insight to the probabilistic mechanism in the coder of LDM. The argument $c z_{t_{i-1}}-x_{t_{i-1}} e^{-\mu\left(t_{i}-t_{i-1}\right)}$ in $\operatorname{erfc}(\cdot)$ is nothing else but the difference between the predicted value of $x_{t_{i}}$ and the predicted value of $z_{t_{i}}$ given the information $\mathcal{F}_{t_{i-1}}$. We shall hence call the functional $\mathbf{F}_{t_{i}}(w)$ defined in (3.14) the predicted-error transform. 


\section{The absolute continuity of the distribution induced by the predicted-error transform}

We shall now prove that the random variable $\mathbf{F}_{t_{i}}(w)$ in (3.14) induces a probability distribution with a density w.r.t. the Lebesgue measure. Since $\mathbf{F}_{t_{i}}(w)$ is another functional of the Wiener process $w^{t_{i-1}}=\left(w_{t}, \mathcal{F}_{t}\right) \quad 0 \leq t \leq t_{i-1}$, this proof is appropriately done as an application of Malliavin's calculus (in the form elaborated in [12] and [16]).

$\mathbf{F}_{t_{i}}(w)$ is a square integrable $\left(E\left|\mathbf{F}_{t_{i}}(w)\right|^{2}<\infty\right)$ functional of $w^{t_{i-1}}$. Let us suppose that $u=\left\{u_{s} \mid 0 \leq s \leq t_{i-1}\right\}$ is a random process on $(\Omega, F, P)$ with Lebesgue measurable and square integrable sample paths. We shall in the sequel select $u$ so that any $u_{s}$ will additionally be adapted to $\mathcal{F}_{s}$, although the work of Zakai and Nualart [12] shows that this is not necessary.

It will first be proved that the directional derivative of $\mathbf{F}_{t_{i}}(w)$ in the $\int u_{s} d s$ direction defined as

$$
D_{u} \mathbf{F}_{t_{i}}(w)=\left.\frac{\partial}{\partial \varepsilon} \mathbf{F}_{t_{i}}\left(w+\varepsilon \int u_{s} d s\right)\right|_{\varepsilon=0}
$$

will exist almost surely. Then, roughly stated, if we can exhibit a process $u$ such that the requirements above are satisfied and such that

$$
D_{u} \mathbf{F}_{t_{i}}(w) \neq 0 \quad \text { a.s. }
$$

our claim about the existence of the density will be established.

We need first a simple observation about the effects of a particular perturbation on linear delta modulation. Let us set for $\varepsilon>0$

$$
w_{\varepsilon}=\left\{w_{t}+\varepsilon \int_{0}^{t} u_{h} d h \mid t \geq 0\right\} .
$$

4.1. Lemma. Let $z_{t_{i}}^{\varepsilon}$ and $z_{t_{i}}$ designate the decoded variables corresponding to the $L D M$ of the processes $w_{\varepsilon}$ and $w$, respectively. Then there exists a positive number $\varepsilon_{t_{i}}$ such that $z_{t_{i}}^{\varepsilon}$ and $z_{t_{i}}$ designate the decoded variables corresponding to the $L D M$ of the processes $w_{\varepsilon}$ and $w$, respectively. Then there exists a positive number $\varepsilon_{t_{i}}$ such that

$$
z_{t_{i}}^{\varepsilon}=z_{t_{i}} \quad P-\text { a.s. }
$$

for every $\varepsilon \leq \varepsilon_{t_{i}}$ (or a modification of the process $z$ such that (4.3) holds).

Proof. We shall proceed inductively using (2.3). Since

$$
\begin{aligned}
& z_{0}^{\varepsilon}=z_{0}=w_{0}=0, \\
& z_{t_{1}}^{\varepsilon}=\operatorname{sgn}\left(w_{t_{1}}+\varepsilon \int_{0}^{t_{1}} u_{s} d s\right), \\
& z_{t_{1}}=\operatorname{sgn}\left(w_{t_{1}}\right)
\end{aligned}
$$


The equality (4.3) holds obviously for $\varepsilon \leq \varepsilon_{t_{1}}$ with some $\varepsilon_{t_{1}}$, with the exception of the event $w_{t_{1}}=0$, the probability of which is zero, since $w_{t_{1}}$ is continuously distributed. (Hence we can modify the variable $z_{t_{1}}$ so that it has a value different from zero for all $\omega \in \Omega$.)

Assume now that $\varepsilon_{t_{i-1}}$ has been established so that $z_{t_{i-1}}^{\varepsilon}=z_{t_{i-1}}$ almost surely for $\varepsilon \leq \varepsilon_{t_{i-1}}$.

Then for $\varepsilon \leq \varepsilon_{t_{i-1}}$

$$
\begin{aligned}
z_{t_{i}}^{\varepsilon}-z_{t_{i}} & =c\left(z_{t_{i-1}}^{\varepsilon}-z_{t_{i-1}}\right)+d\left[\operatorname{sgn}\left(w_{t_{i}}^{\varepsilon}-c z_{t_{i-1}}^{\varepsilon}\right)-\operatorname{sgn}\left(w_{t_{i}}-c z_{t_{i-1}}\right)\right] \\
& =d\left[\operatorname{sgn}\left(w_{t_{i}}^{\varepsilon}-c z_{t_{i-1}}^{\varepsilon}\right)-\operatorname{sgn}\left(w_{t_{i}}-c z_{t_{i-1}}\right)\right] .
\end{aligned}
$$

By the same argument as above we can find $\varepsilon_{t_{i}} \leq \varepsilon_{t_{i-1}}$ such that (4.3) holds for $\varepsilon \leq \varepsilon_{t_{i}}$.

Note that the finite union of exceptional sets, where the equalities (4.3) do not hold, has measure zero, and that there is no difficulty in modifying $z$ in the way desired.

The result of the lemma is intuitively obvious in saying that for sufficiently small $\varepsilon$ the process $z^{\varepsilon}$ has on any finite interval paths that are identical with the paths of a modification of $z$. (Note that the number of paths is finite.)

\subsection{Lemma.}

$$
D_{u}\left[\mathbf{F}_{t_{i}}(w)\right]=V_{x}^{\prime}\left(t_{i-1}, w_{t_{i-1}} ; z_{t_{i-1}}, t_{i}\right) \int_{0}^{t_{i-1}} u_{s} d s
$$

where $\left\{u_{s} \mid s \geq 0\right\}$ has square integrable sample paths.

Proof. By Lemma 4.1, with $\varepsilon \leq \varepsilon_{t_{i}}$,

$$
\begin{aligned}
\mathbf{F}_{t_{i}}\left(w+\varepsilon \int u_{s} d s\right)-\mathbf{F}_{t_{i}}(w)= & \operatorname{erfc}\left(\frac{c z_{t_{i-1}}^{\varepsilon}-w_{t_{i-1}}^{\varepsilon}}{\sqrt{4 \pi\left(t_{i}-t_{i-1}\right)}}\right) \\
& -\operatorname{erfc}\left(\frac{c z_{t_{i-1}}-w_{t_{i-1}}}{\sqrt{4 \pi\left(t_{i}-t_{i-1}\right)}}\right) \\
= & V\left(t_{i-1}, w_{t_{i-1}}^{\varepsilon} ; z_{t_{i-1}}, t_{i}\right)-V\left(t_{i-1}, w_{t_{i}} ; z_{t_{i-1}}, t_{i}\right) .
\end{aligned}
$$

Mean value theorem gives now

$$
=V_{x}^{\prime}\left(t_{i-1}, w_{t_{i-1}}+\varepsilon \Theta \int_{0}^{t_{i-1}} u_{s} d s ; z_{t_{i-1}}, t_{i}\right) \cdot \varepsilon \int_{0}^{t_{i-1}} u_{s} d s
$$

where $0<\Theta<1$. Then it is immediate that

$$
\frac{1}{\varepsilon}\left(\mathbf{F}_{t_{i}}\left(w+\varepsilon \int_{0}^{t_{i-1}} u_{s} d s\right)-\mathbf{F}_{t_{i}}(w)\right) \rightarrow V_{x}^{\prime}\left(t_{i-1}, w_{t_{i-1}} ; z_{t_{i-1}}, t_{i}\right) \int_{0}^{t_{i-1}} u_{s} d s
$$


almost surely, as asserted.

4.3. Proposition. The functional $\mathbf{F}_{t_{i}}(w)$ induces on $[-1,1]$ a probability measure that is absolutely continuous with respect to the Lebesgue measure.

Proof. Set

$$
\left.\left.u_{s}=V_{x}^{\prime}\left(s, w_{s} ; z_{t_{j-1}}, t_{j}\right) \quad \text { for } \quad s \in\right] t_{j-1}, t_{j}\right], \quad j=0, \ldots, i .
$$

Then the sample paths of $u=\left\{u_{s} \mid 0 \leq s \leq t_{i-1}\right\}$ are Lebesgue measurable and integrable as well as $\mathcal{F}_{s}$-adapted. Also $D_{u}\left(D_{u}\left[\mathbf{F}_{t_{i}}(w)\right]\right)$ exists by an argument similar to that used in the proof of lemma 4.2.

But then

$$
D_{u} \mathbf{F}_{t_{i}}(w)=V_{x}^{\prime}\left(t_{i-1}, w_{t_{i-1}} ; z_{t_{j-1}}, t_{i}\right) \int_{0}^{t_{i-1}} u_{s} d s>0
$$

almost surely, since $V_{x}^{\prime}\left(t_{i-1}, w_{t_{i-1}} ; z_{t_{i-1}}, t_{i}\right)>0$ for every $\omega$ as is seen by (3.11). Hence the probability law of $\mathbf{F}_{t_{i}}(w)$ possesses a density by Theorem 5.2 of [12] (c.f. Proposition 2.3.1 in [16]).

Acknowledgements. The author is grateful to the Department of Systems Engineering and Mathematics at the Luleå University of Technology for its support of this research. The referees are thanked for their careful reading of the paper.

\section{References}

[1] Berger, T.: Information rates of Wiener process. - IEEE Trans. Inform. Theory IT-16, 1970, 134-139.

[2] ClaRK, J.M.C.: The representation of functionals of Brownian motion by stochastic integrals. - Ann. Math. Statist. 41, 1970, 1282-1295.

[3] DAvis, M.H.A.: Functionals of diffusion processes as stochastic integrals. - Math. Proc. Cambridge Philos. Soc. 87, 1980, 157-166.

[4] Edgar, G.A., and L. Sucheston: Amarts: A class of asymptotic martingales A: Discrete parameter. - Multivariate Anal. 6, 1976, 193-221.

[5] Gabor, G., and Z. Györfi: Recursive source coding. - Springer Verlag, New York, 1986.

[6] Gersho, A.: Stochastic stability of delta modulation. - Bell System Tech. J. 51, 1972, 821-841.

[7] Hall, P., and C.C. Heyde: Martingale limit theory and its application. - Academic Press, New York, 1980.

[8] JAYANT, N.S.: Adaptive delta modulation with a one-bit memory. - Bell System Tech. J. $49,1970,321-342$.

[9] KosKI, T.: Stochastic stability of delta modulator (I). - Report 7-88 Dept. Math. LuTH, Luleå, 1988.

[10] Liptser, R.Sh., and A.N. Shiryayev: Statistics of Random Processes, I. - SpringerVerlag, Berlin, 1977.

[11] Masry, E., and S. Cambanis: Delta modulation of the Wiener process. - IEEE Trans. Comm. 23, 1975, 1257-1300. 
[12] NUALART, D., and M. ZAKAI: Generalized stochastic integrals and the Malliavin calculus. - Probab. Theory Related Fields 73, 1986, 255-280.

[13] Slepian, D.: On Delta modulation. - Bell System Tech. J. 51, 1972, 2101-2137.

[14] Steele, R.: Delta modulation systems. - Pentech Press, London, 1975.

[15] Widder, D.V.: The heat equation. - Academic Press, London, 1975.

[16] ZAKAI, M.: The Malliavin calculus. - Acta Appl. Math. 3, 1985, 175-207.

[17] Zetterberg, L.H.: A comparison between delta and pulse code modulation. - Ericsson Technics 11, 1955, 95-179.

Luleå University of Technology

Department of Systems Engineering and Mathematics

S-951 87 Luleå

Sweden 